\title{
The use of midazolam and flumazenil for invasive radiographic procedures
}

\author{
S.P. Roberts, J. Hargreaves and B.J. Pollard \\ University Department of Anaesthesia, University Hospital of South Manchester, Nell Lane, West \\ Didsbury, Manchester M20 8LR, UK
}

Summary: This study has examined the use of flumazenil to improve recovery following sedation with midazolam in elderly patients undergoing invasive radiological procedures. Forty patients received either flumazenil or placebo in a randomized double-blind fashion following midazolam sedation. Nalbuphine 10-15 mg was given for premedication. All but one of the patients in the flumazenil group were fully awake immediately following the reversal agents compared to only one in the placebo group $(P=0.016)$. This statistically significant difference remained after 20 minutes $(P=0.029)$. There were no adverse effects on heart rate, blood pressure, forced expiratory volume in one second, vital capacity or oxygen saturation. The majority of patients $(\mathbf{7 8 \%}$ overall) could not recall their procedure. All patients said that they would be willing to undergo a similar technique in the future.

\section{Introduction}

The provision of safe and acceptable conditions for invasive radiographic procedures can present difficulties. General anaesthesia may be performed, although a combination of local infiltration analgesia and sedation is now commonly used. The sedation is usually provided by either a combination of an opioid analgesic with a major tranquillizer or an intravenous benzodiazepine. Prolonged sedation is undesirable, particularly in the elderly patient, and it would be advantageous if the effect of sedation could be easily and safely reversed.

Patients undergoing invasive radiographic procedures often have severe cardiovascular and respiratory disease and any technique employed for the sedation of these patients should not cause adverse haemodynamic or respiratory effects. An early study by Ricou et al. ${ }^{1}$ has shown that the benzodiazepine antagonist flumazenil is suitable for reversing the sedative effects of midazolam in the elderly patient. It has also been suggested that flumazenil may improve respiratory function ${ }^{2}$ following sedation with midazolam and that it produces stable cardiovascular conditions. ${ }^{3}$ It was decided therefore to investigate the use of flumazenil to improve recovery following sedation with midazolam in elderly patients undergoing invasive radiological procedures.

Correspondence: B.J. Pollard, B.Pharm., M.D., F.R.C.A., University Department of Anaesthesia, Manchester Royal Infirmary, Oxford Road, Manchester M13 9WL, UK. Accepted: 19 July 1993

\section{Materials and methods}

Approval was obtained from the hospital ethie committee and informed consent sought from eash $\mathrm{h}$ subject. Forty patients were randomized to rece either flumazenil or placebo following midazolam乏 sedation in a double-blind parallel group design. Patients under 50 years of age were excluded from the trial, as were patients sensitive to benzo- $\frac{\mathbb{Q}}{\mathbb{Q}}$ diazepines or receiving chronic benzodiazepine therapy. Chronic renal or hepatic impairment also윽 excluded patients from participating in the study.

The patients' weight, age, sex, drug history and past medical history were recorded and their ASA status noted. An intramuscular premedication of 3 the opioid analgesic nalbuphine was prescribed in adose of $10 \mathrm{mg}$ for patients less than $60 \mathrm{~kg}$ and 3 . $15 \mathrm{mg}$ for those greater than $60 \mathrm{~kg}$. This was given approximately one hour pre-operatively.

On arrival in the X-ray department, the follow-o ing parameters were recorded: blood pressure, pulse rate, vital capacity, forced expiratory volumeo in one second $\left(\mathrm{FEV}_{1}\right)$, and oxygen saturation (pulse oximeter). The Maddox Wing test (see below) was $\circ$ performed and a memory card shown to the N patient. An intravenous cannula was inserted into the patient's hand and midazolam was given slowlyo until the patient became drowsy. The dose was titrated so that communication with the patient $\frac{-}{\bar{C}}$ was not lost. The skin was infiltrated with lignocaine prior to catheter insertion. The duration 0 and nature of the procedure were recorded, and the $\overline{0}$ blood pressure and heart rate measured at regular $\stackrel{\mathrm{Q}}{\Omega}$ 
intervals. The electrocardiogram was continually displayed.

On termination of the procedure the blood pressure, heart rate, respiratory rate, end tidal carbon dioxide concentration and oxygen saturation were again noted. The patient was again asked to perform the Maddox Wing test. The degree of sedation was assessed using the scale in Table $I$ and then the contents of the blinded ampoule (either flumazenil $0.5 \mathrm{mg}$ or placebo) was given at a rate of $1 \mathrm{ml}$ every 30 seconds.

At 5 minute intervals following administration of the test medication, the blood pressure, heart rate, respiratory rate, sedation score and Maddox Wing test were again recorded. At the first 5 minute test the end tidal $\mathrm{CO}_{2}$ and oxygen saturation were also measured and at 10 minutes the body temperature, vital capacity and $\mathrm{FEV}_{1}$ were also measured. During the 20 minute assessment period in the recovery room, the patients were questioned concerning recall of the memory card. The incidence of nausea and vomiting was also noted.

Following the procedure, each subject was visited on the ward. Any incidence of thrombophlebitis was noted. Each subject was questioned concerning nausea and vomiting, whether they recalled the procedure and if that recall was pleasant or not. Any adverse event at any time was recorded.

\section{Maddox Wing test}

The physiological position of the eyes at rest is in divergence and normal congruent vision is maintained through an active process. Following general anaesthesia there is an increased tendency to divergence of the eyes and this can be measured in the conscious co-operative subject using the Maddox Wing apparatus. The subject looks through the eyepiece into the instrument. The field of vision is divided by shaped wings such that one eye sees a numbered horizontal scale and the other sees an arrow. Divergence of the eyes causes the arrow to appear to move along the scale and the patient is asked to specifiy to which number the arrow is pointing when it has become stationary. This number is the divergence of the eyes measured with prism dioptres. ${ }^{4}$

Table I Sedation score

\begin{tabular}{ll}
\hline 0 & Asleep, unrousable \\
1 & Asleep, rousable \\
2 & Drowsy and disorientated \\
3 & Awake, not fully orientated \\
4 & Awake and orientated \\
\hline
\end{tabular}

\section{Statistical analysis}

The data were analysed following the advice of a medical statistician. Formal statistical comparison of the two treatment groups was performed on sedation scores at each assessment separately using a chi-squared test of independence. Cardiovascular parameters (systolic blood pressure and heart rate) were analysed using a two-sample $t$-test applied to the maximum change from pre-reversal exhibited by each patient over the post-reversal period. All other treatment group comparisons (demography, timings, doses, respiratory function and Maddox Wing test) were informal, being based on inspection of the appropriate summary statistics.

\section{Results}

The mean age of the patients was 60.9 years (range $50-77$ ) for the saline group and 62.4 years (range $50-77$ ) for the flumazenil group. The mean weights for the two groups were $67.7 \mathrm{~kg}$ (range 42-90) and $68.9 \mathrm{~kg}(45-104)$, respectively. The two groups were well balanced with respect to these characteristics; $70 \%$ of patients were male. All but four patients were ASA grade 3. There was one ASA grade 1 and one ASA grade 2 in the placebo group and one ASA 4 patient in each group. Pre-existing medical conditions for the patients in the two groups are shown in Table II.

Patients underwent a number of different procedures, the commonest of which was peripheral angiography (seven placebo, 11 flumazenil) and aortogram (six placebo, four flumazenil). Other procedures including angioplasty, carotid angiography and renal angiography.

The two groups were well matched in terms of premedication dose of nalbuphine (flumazenil group $0.19 \mathrm{mg} / \mathrm{kg}$, placebo group $0.21 \mathrm{mg} / \mathrm{kg}$ ) which was given at a mean time of 58.8 minutes before the procedure. The procedures lasted approximately 30 minutes (means 32.1 minutes

Table II Co-existing medical disorders of patients

\begin{tabular}{lcc}
\hline & Placebo & Flumazenil \\
\hline Any & 18 & 20 \\
Any cardiovascular & 17 & 20 \\
Peripheral vascular disease & 9 & 15 \\
Ischaemic heart disease & 8 & 4 \\
Cardiac failure & 0 & 1 \\
Hypertension & 2 & 4 \\
Atrial fibrillation & 0 & 1 \\
Recent myocardial infarction & 0 & 1 \\
Transient ischaemic attacks & 7 & 4 \\
Diabetes mellitus & 4 & 1 \\
\hline
\end{tabular}


placebo, 28.5 minutes flumazenil) and the two groups were well matched in terms of dosage of midazolam $(0.06 \mathrm{mg} / \mathrm{kg}$ placebo group, $0.05 \mathrm{mg} /$ $\mathrm{kg}$ flumazenil group). The mean time to the postoperative visit was approximately 5 hours.

Immediately preceding the administration of flumazenil or placebo there was no statistically significant difference between the two groups in terms of sedation score, although the placebo group was slightly more sedated ( 2.7 compared to 3.0 in the flumazenil group $P<0.2$ ). At 5 minutes post-drug the difference between the two groups was marked $(P=0.016)$ with all but one of the flumazenil group being fully awake (the exception had a score of 3). This was in comparison to the placebo group where only $5 \%$ were fully awake. Figure 1 shows the percentage of patients with sedation scores of four at assessment post-reversal. At 20 minutes post-reversal the difference remained $(P=0.029)$ with all flumazenil patients fully awake, but only $70 \%$ of placebo patients fully awake.

A number of patients were unable to perform the Maddox Wing test due to poor eyesight (six in the flumazenil group, three in the placebo group). In addition to this, two patients in the flumazenil group could not perform the pre-reversal test. In the placebo group two patients could not perform the test at pre-reversal and three could not perform the test at post-reversal assessments as well as pre-reversal. The missing data were assigned a value of 25 (the maximum recorded was 22) because it represented 'too sedated' a state. The results of the Maddox Wing test are shown in Figure 2.

A rise in score from baseline to pre-reversal is

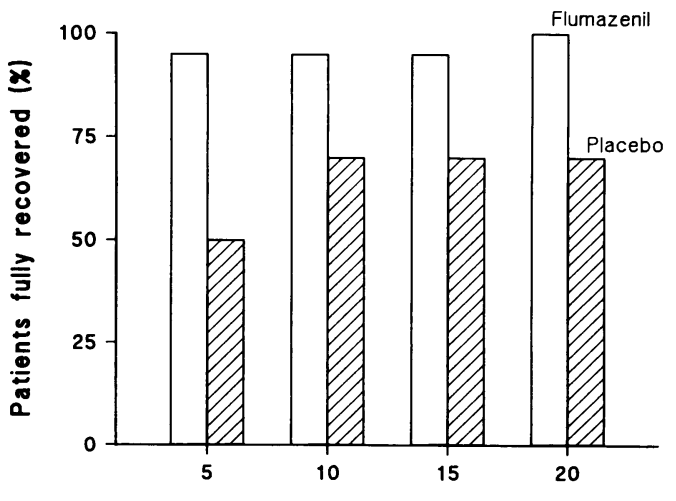

Time after reversal (minutes)

Figure 1 Percentage of patients with sedation score of four at each time point following administration of the reversal medication.

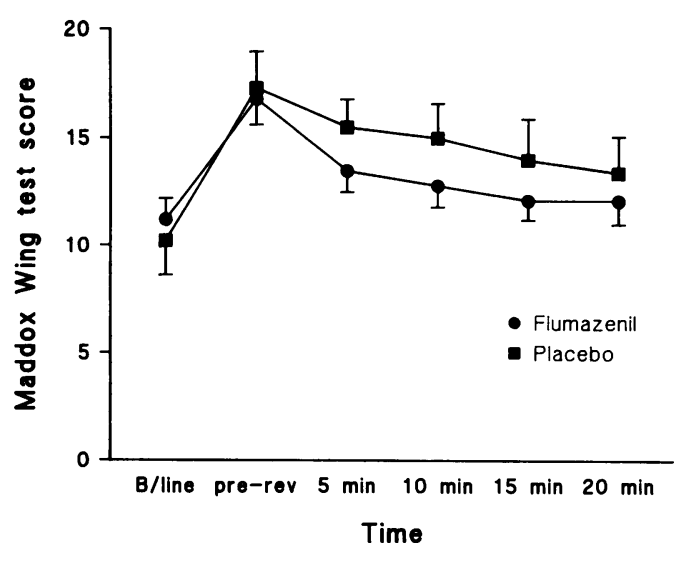

Figure 2 Maddox Wing test scores.

seen, followed by a fall in both groups. The mean ㅇ values are lower in the flumazenil group.

There was little change in oxygen saturation (Table III) either from baseline to pre-reversal or $\overparen{\Phi}$ from pre-reversal to 5 minutes post-reversal in the placebo group. The saturation decreased in the flumazenil group pre-reversal and increased sligh $\mathbb{E}$ ly post-reversal.

The $\mathrm{FEV}_{1}$ and $\mathrm{FVC}$ results do not differ between. pre- and post-reversal values in both groups (Table III) but two patients in the placebo group remained too sedated to perform the tests. The respiratory rate did not differ substantially between the two groups post-reversal.

The systolic blood pressure was very similar in $\overrightarrow{\vec{F}}$ both groups before the procedure (Table IV). It fell $\frac{\mathrm{O}}{3}$ during the procedure, subsequently recovering by the end of the procedure (pre-reversal), although it had not returned to the baseline values. It is possible that the baseline value was elevated above 3 . normal due to anxiety. Following reversal with either flumazenil or placebo there was very little change in the blood pressure (Table IV). Excellent $\delta$ stability of heart rate was seen throughout with no differences between the groups (Table IV).

Nausea and vomiting occurred in one patient in the flumazenil group by the 20 minute assessment. At the post-procedure assessment, one flumazenil patient and three placebo patients complained of $\sigma$ nausea.

The majority of patients could not recall their $\mathrm{N}_{\mathrm{N}}$ procedure ( $80 \%$ placebo, $75 \%$ flumazenil). Of the ten patients (four placebo, six flumazenil) who had some recall of the procedure, only one (placebo) found the recall unpleasant. Only one patient $\stackrel{?}{?}$ (flumazenil) had mild thrombophlebitis. All 0 patients said they would be willing to undergo a similar procedure in the future. 
Table III Respiratory parameters - means (SEM)

\begin{tabular}{lccc}
\hline & Baseline & Pre-reversal & Post-reversal \\
\hline Placebo group & & & \\
$\quad$ Forced expiratory volume (litres) & $1.82(0.15)$ & - & $1.69(0.14)$ \\
Forced vital capacity (litres) & $2.36(0.17)$ & - & $2.13(0.17)$ \\
End tidal $\mathrm{CO}_{2}$ (mmHg) & - & $40.4(1.5)$ & $39.2(1.4)$ \\
Respiration rate (per minute) & - & $14.5(0.8)$ & $15.1(0.8)$ \\
Oxygen saturation (\%) & $92.4(1.1)$ & $92.1(1.1)$ & $91.9(1.0)$ \\
Flumazenil group & & & \\
$\quad$ Forced expiratory volume (l) & $1.53(0.13)$ & - & $1.53(0.12)$ \\
Forced vital capacity (1) & $2.22(0.15)$ & - & $2.11(0.15)$ \\
End tidal $\mathrm{CO}_{2}$ (mmHg) & - & $43.2(1.3)$ & $40.3(1.1)$ \\
Respiration rate (per minute) & - & $14.8(0.7)$ & $14.3(0.6)$ \\
Oxygen saturation (\%) & $92.3(0.7)$ & $89.3(0.9)$ & $90.5(0.9)$ \\
\hline
\end{tabular}

Table IV Systolic blood pressure and heart rate measurements before, during and following the procedures

\begin{tabular}{|c|c|c|c|c|c|c|c|c|}
\hline & Baseline & 5 minutes & 15 minutes & Pre-reversal & 5 minutes & 10 minutes & 15 minutes & 20 minutes \\
\hline \multicolumn{9}{|c|}{ Systolic blood pressure } \\
\hline Placebo & $155(7)$ & $134(7)$ & $125(9)$ & $137(9)$ & $139(8)$ & $139(7)$ & $134(6)$ & $132(7)$ \\
\hline Flumazenil & $158(6)$ & $135(7)$ & $129(4)$ & $135(5)$ & $139(5)$ & $138(4)$ & $137(5)$ & $136(5)$ \\
\hline \multicolumn{9}{|l|}{ Heart rate } \\
\hline Placebo & $79(3)$ & $76(5)$ & $78(5)$ & $78(4)$ & $78(4)$ & $78(4)$ & $76(4)$ & 78 (4) \\
\hline Flumazenil & $79(4)$ & $80(4)$ & $79(4)$ & $77(3)$ & $76(3)$ & $75(4)$ & $72(3)$ & $71(2)$ \\
\hline
\end{tabular}

Data are given as mean (SEM). Units are $\mathrm{mmHg}$ for systolic blood pressure and beats/minute for heart rate.

\section{Discussion}

The study has shown that flumazenil can be used effectively and safely to reverse midazolam sedation in elderly patients with systemic disease. Nearly all of the patients in the study had concomitant disorders; peripheral vascular disease, ischaemic heart disease and cerebrovascular disease were the most common.

All of the patients who took part in the study found the procedure to be satisfactory in that they would accept the same technique again. The reversal of sedation by flumazenil produced dramatic differences between the two groups. At 5 minutes post-reversal all but one of the flumazenil patients were fully awake, that patient being grade 3 , i.e. drowsy and disorientated. This compared to only $50 \%$ of the placebo group being fully awake.

The other measurement of sedation used was the Maddox Wing test. ${ }^{4}$ Although this showed some difference between the groups, the scores being lower in the placebo group, this difference was not great. This may be partially due to the fact that good patient co-operation and eyesight is necessary, and it is possible that it is not a good assessment of sedation in elderly patients.
The difference in degree of sedation between the groups which persisted at the 20 minute assessment is important. The early return of a patient's protective airway reflexes makes that patient less vulnerable to regurgitation and aspiration. Patients can also be returned to the ward sooner, facilitating the conduct of a list of procedures.

Flumazenil has a similar half-life to midazolam ${ }^{5,6}$ so that reversal of a bolus dose can be performed with minimal risk of resedation later. The same would not be true of other benzodiazepines such as diazepam, which have a longer half life.

The safety of any agent is important when considering its clinical applications. In patients with ischaemic heart disease and cerebrovascular disease, haemodynamic stability is essential. A characteristic of this technique is that there was marked stability of pulse and blood pressure post-reversal with flumazenil. This finding is in agreement with previous work. ${ }^{3,7}$ This study shows that those previously reported findings can be extended to the elderly and frail patient.

There was little evidence of significant change in respiratory parameters following administration of flumazenil. Radakovic et $\mathrm{al}^{2}{ }^{2}$ showed that flumazenil significantly improved ventilatory function 
following midazolam, although they used doses which were much greater than those used in the present study ( $15.4 \mathrm{mg}$ compared to $3.8 \mathrm{mg})$. It would appear that flumazenil has no deleterious effect on ventilation function and may improve it when larger doses of midazolam are used. It must be remembered that all patients received nalbuphine as part of their premedicant. This should not have affected any comparison between the two groups because all patients received nalbuphine. Nalbuphine does, however, have a depressant effect on respiration and it may have contributed, at least partly, to the low baseline respiratory parameters and low baseline oxygen saturation.

Ricou et al. ${ }^{1}$ reported a $20 \%$ incidence of anxiety

\section{References}

1. Ricou, B., Forster, A., Bruckner, A., Chastonay, P. \& Gemperle, M. Clinical evaluation of a specific benzodiazepine antagonist (RO 15-1788). Br J Anaes 1986, 58: 1005-1011.

2. Radakovic, D., Toiui, D. \& Bentzinger, C. Double-blind, placebo controlled study of the effects of RO 15-1788 on recovery of ventilatory function after total intravenous anaesthesia with midazolam-alfentanyl. Eur J Anaes 1988, (Suppl 2): 279-282.

3. Marty, J. \& Joyon, D. Haemodynamic responses following reversal of benzodiazepine induced anaesthesia or sedation with flumazenil. Eur J Anaes 1988, (Suppl 2): 167-171.

4. Hannington-Kiff, J.G. Measurement of recovery from outpatient general anaesthesia with a simple ocular test. $\mathrm{Br} \mathrm{Med} \mathrm{J}$ 1970, 3: $132-135$. and feelings of impending death in elderly patients undergoing flumazenil reversal. We found no difference between the two groups with respect of $\propto$ acceptability of the technique. It may be that the addition of an analgesic, e.g. nalbuphine, reduces $\stackrel{\overrightarrow{\vec{\rho}}}{\vec{\theta}}$ the incidence of this side effect.

In conclusion, the use of flumazenil following midazolam sedation provided a safe and effective $\overline{\bar{\rho}}$ technique for invasive radiographic procedures. Patients with severe systemic disease can be safely sedated without adverse cardiovascular or respiratory changes. Flumazenil improved recovery $\vec{O}$ from sedation and the technique was pleasant and acceptable.

5. Roncari, G., Ziegler, W.H. \& Guenhert, T.W. Pharmacokinetics of the new benzodiazepine antagonist RO 15-1788 in N man following intravenous and oral administration. $\mathrm{Br} \mathrm{J} \mathrm{Clin}$ Pharmacol 1986, 22: 421-428.

6. Dundee, J.W., Halliday, N.J., Harper, K.W. \& Brogden, R.N. Midazolam, a review of its pharmacological properties and $\square$ therapeutic use. Drugs 1984, 28: 519.

7. Croughwell, N.D., Reeves, J.G., Will, C.J., Kasson, B.J. \& Hawkins, E. Safety of flumazenil in patients with ischaemic heart disease. Eur J Anaes 1988, (Suppl 2): 177-182. 